\title{
Improved Energy Compensation for Time-of-Flight Mass Spectrometry*
}

\author{
R. T. Short and P. J. Todd \\ Oak Ridge National Laboratory, Oak Ridge, Tennessee, USA
}

\begin{abstract}
A model for improved energy compensation in time-of-flight (TOF) mass spectrometry has been developed and tested. This model includes effects of both the acceleration and drift region on mass resolution for surface desorption TOF mass spectrometers that employ ion mirrors to improve mass resolution. Appropriate placement of an additional stage onto the conventional one- and two-stage mirrors provides compensation for flight time spreads, caused by initial ion kinetic energy distributions, in both regions. Experimental results that validate the model calculations are presented for a modified commercial two-stage ion mirror. For example, $m / \Delta m$ for $\mathrm{Na}^{+}$was improved from $\sim 100$ to $\sim 200$ using only a 200-VV drift energy and a 58-cm drift path. (J Am Soc Mass Spectrom 1994, 5, 779-787)
\end{abstract}

$\mathrm{M}$ ass resolution of time-of-flight (TOF) mass spectrometers is limited by the temporal distribution of the analyzed ions at the detector. This temporal distribution is largely a function of the initial temporal, spatial, and velocity distributions of the ions. The initial temporal distribution arises from the finite duration of the ionization pulse, but can be made nearly negligible by using a very short ionization pulse (e.g., several nanoseconds to subnanoseconds). Initial spatial distribution along the axis of the TOF mass spectrometer can be virtually eliminated by creating and ejecting the ions from a plane, as with secondary ionization mass spectrometry (SIMS) [1-3], laser desorption ionization (LDI) [4], matrix-assisted laser desorption ionization (MALDI) [5, 6], and plasma desorption sources [7-9]. It is also possible to minimize the effects of initial spatial distributions from gas-phase sources by proper choice of ion acceleration fields and analyzer geometry, as shown by Wiley and McLaren [10]. The initial ion velocity distribution then becomes the dominant initial distribution that limits mass resolution of time-of-flight mass spectrometers. In fact, this feature has been exploited to obtain measurements of mean ion kinetic energies in gas phase sources for various ionization methods [11, 12].

Effects of the initial ion velocity (or kinetic energy) distribution on TOF mass resolulion also have been significantly reduced with the introduction of the electrostatic ion mirror, or reflectron [13-15]. Ion mirrors work on the principle that faster ions penetrate deeper

Address reprint requests to R. T. Short, P.O. Box 2008, Buildiny 5510, MS-6365, Oak Ridge National Laboratory, Oak Ridge, TN 37831-6365.

* The submitted manuscript has been authored by a contractor of the U.S. Government under contract No. DE-AC05-84OR21400. Accordingly, the U.S. Government retains a nonexclusive, royalty-free license to publish or reproduce the published form of the contribution, or allow others to do so, for U.S. Government purposes. into the mirror and consequently spend more time in the mirror. This extra time in the mirror compensates for the shorter flight times of faster ions in the field-free drift region. With the proper choice of geometry and mirror electric field(s), the flight times of all ions of a common mass-to-charge ratio through the drift region and mirror can be made to be independent of initial kinetic energy to first-order approximation for a onestage ion mirror [16] and to second-order approximation for a two-stage mirror [13]. Such analysis assumes that all ions start from a well defined plane and stop at a well defined detection plane, which are both perpendicular to the TOF spectrometer axis. Mass resolution in excess of $m / \Delta m=10,000$ has been demonstrated by using ion mirrors of these types in several labs [9, 16-19].

Gridless electrostatic ion mirrors that improve ion transmission also have been developed and are used by a number of groups $[1,20-22]$. Gridless mirrors, however, are difficult to describe analytically [23]; that is, no mathematical expression can be used to explicitly describe ion trajectories. Gridless mirrors are optimized by using complex ray tracing programs and often require extensive empirical tuning. Ion mirrors that employ grids to define electric field regions are simpler to design and consequently see much wider use in the mass spectrometry community.

Various dynamic methods have been proposed [24, $25]$ and used [10, 26, 27] as well to compensate for temporal, spatial, and kinetic energy spreads. Improvement in mass resolution for these dynamic techniques, however, is mass dependent; mass resolution is improved in one region of the mass spectrum at the cost of worsened mass resolution in the other regions. The present work deals only with electrostatic approaches (which are not mass dependent) to improve TOF mass resolution. 
Although the one-stage and two-stage ion mirrors have been shown analytically to compensate for flight time spreads that arise from kinetic energy spreads in the field-free drift region, they do not compensate for corresponding flight time variations in the acceleration region. Ions must be accelerated from the sample surface, however, in order to separate them by their mass-to-charge ratio using TOF. Although the time spent in the acceleration region is typically small compared to the flight time in the rest of the TOF mass spectrometer, the time spread in this region can become the major limiting factor in mass resolution, as recently pointed out by Standing et al. [28]. In fact, this contribution is often greater than second-order contributions from the field-free drift region. This has led many groups to return to use of a one-stage mirror, foregoing the added complication of the two-stage mirror, because the theoretical increase in mass resolution is overshadowed by time spreads in the acceleration region.

Previous strategies to minimize the loss of mass resolution from the acceleration region have been limited mainly to reducing the relative time that the ions spend in the acceleration region. This is accomplished by using high acceleration voltages and small extraction distances. While this approach has proved sufficient in many cases, it is not a complete solution to the problem, because it imposes severe restrictions on TOF ion source and mass spectrometer design.

A large extraction distance in the ion source region is desirable for several reasons. There is more flexibility for introduction of an ionizing beam (e.g., primary ion beam or laser beam) if the extraction grid is farther from the sample surface. Wide-angle imaging or array analysis by rastering a focused ion beam becomes possible with a large extraction distance, as demonstrated with a quadrupole-based SIMS microprobe developed in this lab [29]. In addition, memory effects from inadvertent sample sputtering onto the extraction grid become less of a problem with an extended extraction ficld. Unless flight time spreads in the acceleration region can be analyzed and a suitable compensation scheme developed, the advantages of a large extraction distance are accompanied by a significant loss of mass resolution.

Historically, longer flight tubes have been employed to enhance mass resolution, because mass resolution in TOF mass spectrometry can be improved by increasing ion flight times if the ion peak widths do not broaden correspondingly. An alternative method to increase ion flight times would be to slow the ions down, that is, use a lower accelerating voltage. This approach could lead to much more rompact high resolution TOF mass spectrometers. Unless flight time spreads in the acceleration region are directly compensated, however, ion peaks broaden with a reduction in accelerating voltage, often yielding a reduction in mass resolution.

To address the problem of flight time spreads arising from ion kinetic energy distributions in the acceler- ation region, we developed a theoretical basis for modification of the conventional one- and two-stage ion mirrors. The theory demonstrates analytically that energy compensation can be accomplished, not only for the field-free drift region, but for the acceleration region as well, by using the modified one-stage and two-stage mirrors. Unbeknownst to us, Vestal [30] independently derived equivalent equations and reported on similar modifications to conventional ion mirrors [31]. The significance of these analyses is sufficient to warrant experimental verification, which is presented here. To test the theory, we have modified a commercially available two-stage ion mirror. Our first experimental results from a SIMS TOF system are discussed below. These results demonstrate the ability to achieve energy compensation throughout TOF mass spectrometers that use surface desorption ion sources, such as SIMS, LDI, MALDI, or plasma desorption.

\section{Theory}

\section{One-Stage Mirror}

Equations that describe the flight times of ions in a TOF spectrometer with a one-stage electrostatic mirror have been derived elsewhere [16]. They will be summarized here with notation consistent with further development in this paper. Figure 1a schematically shows [32] the flight path of an ion in a one-stage reflecting TOF mass spectrometer. The total flight time $(t)$ of an ion with mass $(m)$, charge $(q)$, and drift velocity $(v)$ along the spectrometer $(z)$ axis can be expressed as the sum of flight times in the drift region $\left(t_{\mathrm{L}}\right)$ and mirror $\left(t_{\mathrm{M}}\right)$ :

$$
t=t_{\mathrm{L}}+t_{\mathrm{M}}=\frac{L}{V}+\frac{2 m}{q E_{\mathrm{M}}} v
$$

where $L$ is the total length of the drift path and $E_{M}$ is the mirror electric field. This equation can be rewritten following the format used by Karataev et al. [13] to describe a two-stage mirror:

$$
t=C F(k)
$$

where

$$
\begin{gathered}
C=\sqrt{\frac{m}{2 q U_{\mathrm{o}}}} \\
F(k)=\frac{L}{\sqrt{k}}+\frac{4 l_{\mathrm{o}}}{E_{M}} \sqrt{k}
\end{gathered}
$$

The term $q U_{\mathrm{o}}$ is the ion drift energy corresponding to the axial velocity $(v)$ for an ion with initial energy $q U_{i}=0$. The factor $k$ has a value typically near unity and is defined as

$$
k=\frac{q U}{q U_{\mathrm{o}}}
$$


a
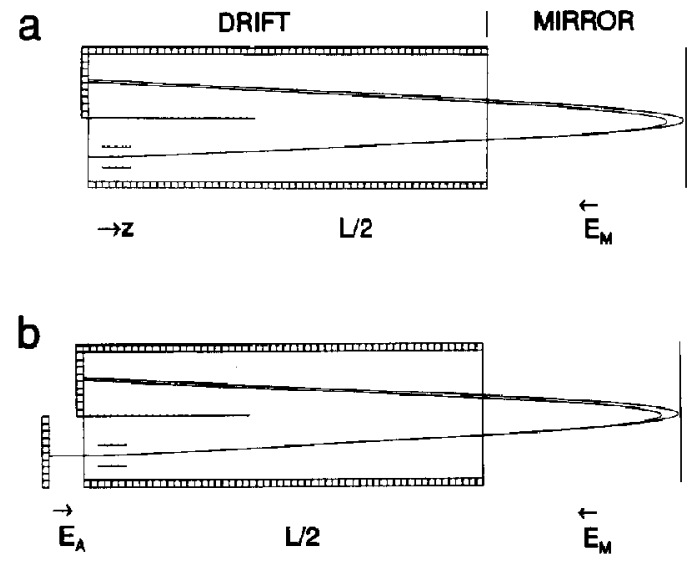

$\mathbf{C}$

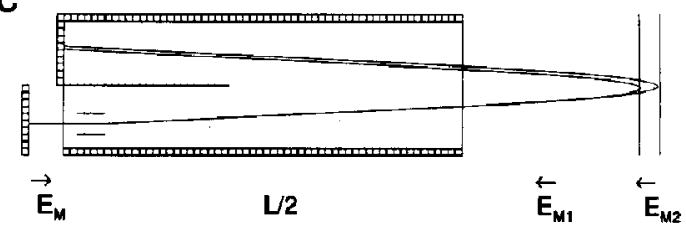

Figure 1. Simulated ion trajectories in a TOF mass spectrometer. (a) Flight paths of two ions in the drift region and one-stage ion mirror. The more energetic ion penetrates deeper into the mirror. Deflection plates are used to deflect the ions at a slight angle into the mirror. (b) Flight paths of ions in a one-stage mirror TOF mass spectrometer, including acceleration from the sample surface. (c) Flight paths of ions in a mass spectrometer with a new two-stage ion mirror. The second stage is placed such that only ions with nonzero initial kinetic energy penetrate $\mathbf{E}_{\mathrm{M} 2}$.

where $q L I$ is the corresponding drift energy of an ion with arbitrary initial energy $q U_{i}$ and can be expressed as

$$
q U=q\left(U_{0}+U_{i}\right)
$$

The following conditions must be satisfied for first-order temporal focusing with respect to ion kinetic energy:

$$
\begin{gathered}
\left.\left(\frac{\partial t}{\partial U}\right)\right|_{U_{1}=0}=0 \\
\left.\left(\frac{\partial F}{\partial k}\right)\right|_{k=1}=0
\end{gathered}
$$

Solving eq 7 by using eq 4 yields the condition

$$
E_{\mathbf{M}}=\frac{4 U_{0}}{L}
$$

Equation 8 defines the mirror electric field required for the flight time $(t)$ of an ion with mass $(m)$ and charge (q) to be independent of initial kinetic energy to a first-order approximation.
If the flight time $\left(t_{A}\right)$ in the acceleration region is included as shown in Figure 1b, eqs 1 and 4 become

$$
\begin{gathered}
t=t_{\mathrm{A}}+t_{\mathrm{L}}+t_{\mathrm{M}} \\
F(k)=\frac{2 U_{\mathrm{o}}}{E_{\mathrm{A}}}(\sqrt{k}-\sqrt{k-1})+\frac{L}{\sqrt{k}}+\frac{4 U_{\mathrm{o}}}{E_{\mathrm{M}}} \sqrt{k}
\end{gathered}
$$

and eq 7 yields

$$
\begin{aligned}
\left.\left(\frac{\partial F}{\partial k}\right)\right|_{k=1} & =\frac{U_{\mathrm{o}}}{E_{\mathrm{A}}}\left(\frac{1}{\sqrt{k}}-\frac{1}{\sqrt{k-1}}\right) \\
-\frac{L}{2 k^{3 / 2}}+\frac{2 U_{\mathrm{o}}}{E_{\mathrm{M}} \sqrt{k}} & =0
\end{aligned}
$$

which is singular at $k=1$, or $U_{\mathrm{i}}=0$; that is, the equation cannot be satisfied for finite values of $U_{0}$ and $E_{\mathrm{A}}$. Consequently, a one-stage ion mirror cannot compensate for an initial ion kinetic energy distribution which extends to $q U_{\mathrm{i}}=0$, if the acceleration region is explicitly included.

\section{New Two-Stage Mirror}

If a second stage (i.e., a grid separating two electric field regions) is added, such that only ions with initial energy $q U_{i}>0$ penetrate into the second stage (Figure 1c), then eqs 9 and 10 become

$$
\begin{gathered}
t=t_{\mathrm{A}}+t_{\mathrm{I}}+t_{\mathrm{M} 1}+t_{\mathrm{M} 2} \\
F(k)=\frac{2 U_{\mathrm{o}}}{E_{\mathrm{A}}}(\sqrt{k}-\sqrt{k-1})+\frac{L}{\sqrt{k}} \\
+\frac{4 U_{\mathrm{o}}}{E_{\mathrm{M} 1}}(\sqrt{k}-\sqrt{k-1})+\frac{4 U_{\mathrm{o}}}{E_{\mathrm{M} 2}} \sqrt{k-1}
\end{gathered}
$$

Note that this geometry is different from the conventional two-stage mirror introduced by Karataev et al. [13] (discussed in more detail in the following section). Solving eq 7 for first-order temporal focusing yields two new conditions:

$$
\begin{gathered}
E_{\mathrm{M} 1}=\frac{4 U_{\mathrm{o}}}{L-2 d_{\mathrm{A}}} \\
E_{\mathrm{M} 2}=\frac{E_{\mathrm{M} 1}}{1+E_{\mathrm{M} 1} / 2 E_{\mathrm{A}}}
\end{gathered}
$$

Equation 14 converges to eq 8 as the acceleration distance $\left(d_{\mathrm{A}}\right)$ goes to zero. This is the mathematical justification for use of a single-stage mirror and very short extraction distance. Equation 15 defines the electric field of the second ion mirror stage that is required to achieve first-order focus, as a function of the ion source acceleration field and the mirror first-stage electric field. If the acceleration field $\left(E_{\mathrm{A}}\right)$ is very large, then $E_{\mathrm{M} 1}$ and $E_{\mathrm{M} 2}$ have essentially the same value. This is consistent with the good mass resolution obtainable with a one-stage mirror and a strong acceleration field. 
Theoretical performance of the ion mirrors for each of the three configurations shown in Figure 1 has been evaluated. Calculated relative flight time spreads versus initial kinetic energy for $\mathrm{TOF}$ mass spectrometer parameters listed in Table 1 are shown in Figure 2. Each plot in Figure 2 represents the calculated flight time difference in each region of the TOF mass spectrometer (relative to the total flight time $t_{0}$ of an ion with $q U_{\mathrm{i}}=0$ ) as a function of initial kinetic energy (relative to the drift energy $q U_{\mathrm{b}}$ of an ion with $q U_{\mathrm{i}}=0$ ). Because mass resolution is defined as

$$
\frac{m}{d m}=\frac{t_{\mathrm{o}}}{2 d t}
$$

a value of $d t / t_{0}=0.01$ on these plots corresponds to a mass resolution limil of $\mathrm{m} / \mathrm{d} m=50$.

Demonstrated in Figure $2 a$ is the predicted energy compensation for a one-stage ion mirror if the flight time in the acceleration region is neglected (Figure 1a). The excess flight time in the mirror has the same energy dependence as the time deficit in the drift region, so the total time difference is very nearly zero - even for initial energies up to $10 \%$ of the drift energy. In Figure $2 b$ are similar plots for the configuration shown in Figure $1 b$, where the flight time in the acceleration region is included in the calculation. The flight time in the mirror is the same as in Figure $2 a$, but the curve for the combined flight time in the acceleration region and drift region has a very different and nonlinear energy dependence. The resulting total flight time curve deviates from zero substantially, even for small values of $U_{i} / U_{0}$. Figure $2 \mathrm{c}$ represents similar calculations for the new two-stage mirror configuration shown in Figure 1c. The excess flight time in the new two-stage mirror has the same functional dependence on initial energy as the time deficit in the combined acceleration and drift regions. Consequently, the total flight time spread is very near zero for initial energies up to $10 \%$ of the nominal drift energy.

Figure 3 shows the total flight time curves from Figure $2 b$ and $c$ together in greater detail for comparison. Limits on mass resolution for the two configurations can be calculated for a particular value of $U_{\mathrm{i}} / U_{\mathrm{o}}$ by using eq 16 and the maximum $d t$ for a particular section of each curve. For example, an initial energy

Table 1. Theoretical TOF mass spectrometer parameters

\begin{tabular}{lll}
\hline Parameter & \multicolumn{1}{c}{ Description } & \multicolumn{1}{c}{ Value } \\
\hline \hline$d_{\mathrm{A}}$ & Acceleration length & $2.50 \mathrm{~cm}$ \\
$L$ & Drift length & $58.0 \mathrm{~cm}$ \\
$q U_{0}$ & Drift energy $\left(U_{\mathrm{i}}=0\right)$ & $2000 \mathrm{eV}$ \\
$E_{\mathrm{M}}$ & One-stage mirror field & $138 \mathrm{~V} / \mathrm{cm}$ \\
$E_{\mathrm{M} 1}$ & Two-stage mirror field $(1)^{\mathrm{b}}$ & $151 \mathrm{~V} / \mathrm{cm}$ \\
$E_{\mathrm{M} 2}$ & Two-stage mirror field $(I)^{\mathrm{b}}$ & $138 \mathrm{~V} / \mathrm{cm}$ \\
\hline
\end{tabular}

${ }^{a}$ Used for Figure 2a and $b$

'Used for Figure 2c only.
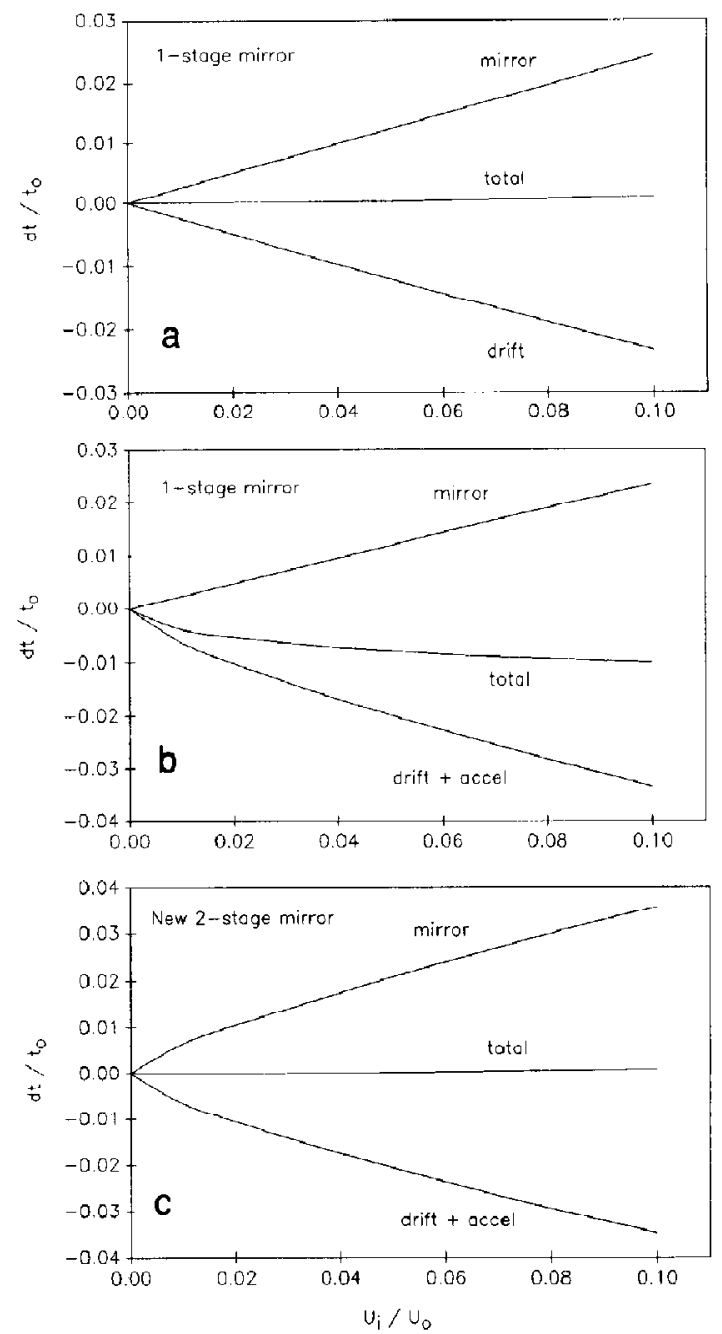

Figure 2. Calculated ion flight time differences $(d t)$ as a function of initial energy $\left(U_{\mathrm{j}}\right)$. (a) In the drift region, the one-stage mirror and total path shown in Figure 1 a. (b) In the acceleration and drift regions, the one-stage mirror and total path shown in Figure $1 b$. (c) In the acceleration and drift regions, the new two-stage mirror and total path shown in Figure 1c. Values of $d t$ are plotted relative to the total flight time $\left(t_{\mathrm{o}}\right)$ of an ion with $U_{\mathrm{i}}=0$, and values of $U_{\mathrm{i}}$ are plotted relative to the nominal drift energy $U_{13}$.

spread of $5 \%$ of the drift energy (i.e., $U_{\mathrm{i}} / U_{\mathrm{o}}=0.05$ ) limits the mass resolution of the one-stage configuration to $m / d m=63$. The new two-stage configuration, however, has a limiting mass resolution of $m / d m=$ 3670. It has been shown [33] that mass resolution for the one-stage configuration can be improved (for the combined acceleration and drift regions) by reducing the mirror field from the optimum value determined in eq 8 . The third curve in Figure 3 represents the total flight time curve for a reduced mirror electric field that is $92 \mathrm{~V} / \mathrm{cm}$ (i.e., $66.7 \%$ of the value listed in Table 1 ). 
The mass resolution calculated for this case is $m / d m=207$ : only a net improvement of a factor of 3 . Consequently, the new two-stage mirror configuration can potentially improve mass resolution by more than order of magnitude for ion sources with a relatively large extraction distance or weak extraction field. If a very short extraction distance and very strong extraction field are used, as is typical in many SIMS experiments, $U_{i} / U_{0}$ is typically on the order of 0.001 and the scales in Figures 2 and 3 are not particularly relevant. In these cases, improvement in mass resolution may be limited by other factors such as mechanical tolerances, fringe fields, and grid effects. A thorough discussion of each of these effects can be found in a review by Boesl et al. [34].

\section{A Three-Stage Mirror}

The second-order temporal focusing condition for a one-stage mirror,

$$
\begin{aligned}
\left.\left(\frac{\partial^{2} t}{\partial U^{2}}\right)\right|_{U_{1}=0} & =0 \\
\left.\left(\frac{\partial^{2} F}{\partial k^{2}}\right)\right|_{k=1} & =0
\end{aligned}
$$

gives the following condition (neglecting flight time in the acceleration region):

$$
E_{\mathrm{M}}=\frac{4 U_{\mathrm{o}}}{3 L}
$$

Note that for a one-stage ion mirror, except for trivial solutions, there is no value of $E_{\mathrm{M}}$ that satisfies eqs 8 and 18 simultaneously. As for the one-stage mirror, the second-order focusing condition for the new configuration of a two-stage mirror (described above) cannot be satisfied simultaneously with the first-order condition. Karataev et al. [13], however, demonstrated that use of a two-stage ion mirror with the configuration shown in Figure 4a (which we refer to as a conven-

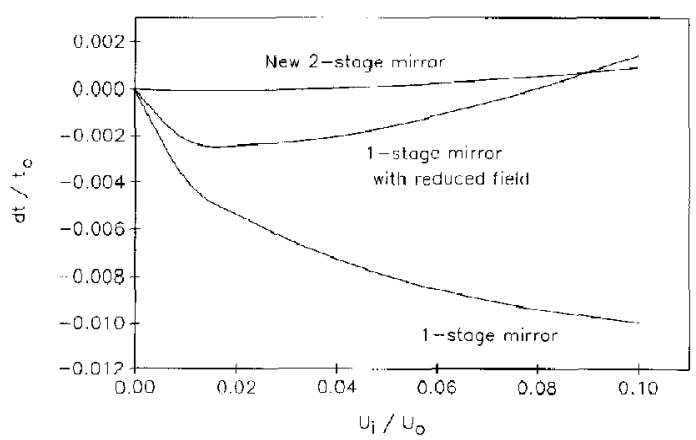

Figure 3. Comparison of one-stage and new two-stage mirrors. Total relative ion flight time differences $\left(d t / t_{0}\right)$ for ions as a function of relative initial kinetic energy $\left(U_{\mathrm{i}} / U_{\mathrm{o}}\right)$.
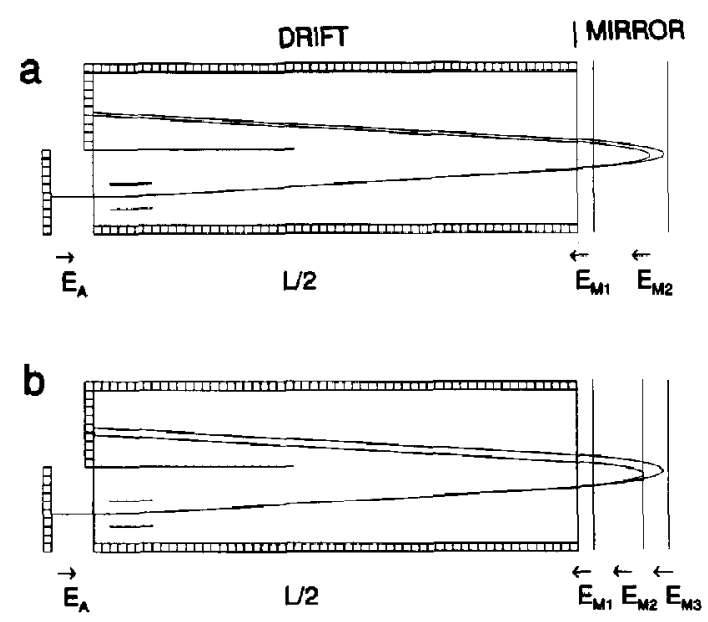

Figure 4. Simulated ion trajectories in a TOF mass spectrometer (a) with a conventional two-stage ion mirror and (b) with a new three-stage ion mirror.

tional two-stage mirror) provides energy compensation to both first- and second-order approximations for flight time spreads in the field-free drift region. In the conventional two-stage ion mirror, ions are decelerated to approximately $1 / 3$ of their drift energy $\left(U_{0}\right)$ in the first stage and then are reflected in the second stage. The conventional two-stage mirror, however, does not compensate for flight time spreads in the acceleration region. Calculations of flight times in the conventional two-stage mirror and combined acceleration and drift regions yield results similar to those shown in Figure $2 b$ for the one-stage mirror.

By using an approach that is similar to the theoretical development for modification of the one-stage ion mirror, we and Vestal [30] independently derived ion equations of motion in a TOF mass spectrometer that include consideration of the acceleration region and $a$ modified two-stage ion mirror (i.e., a three-stage mirror). With the appropriate configuration, a three-stage mirror provides first- and second-order energy compensation for flight time spreads in both the acceleration and drift regions. Figure $4 \mathrm{~b}$ represents the flight path of an ion in a TOF mass spectrometer with an acceleration region and a three-stage mirror. As with the new two-stage design, the terminal third stage is placed such that only ions with nonzero initial kinetic energy $\left(q U_{i}=0\right)$ enter this region. Two of the necessary focusing conditions for the three-stage mirror (the values of electric fields in the first two stages of the mirror) remain essentially those determined by Karataev et al. [13], as long as the drift path (L) remains much larger than the dimensions of the first mirror stage $\left(d_{M 1}\right)$ and acceleration region $\left(d_{A}\right)$. The third condition for the three-stage mirror,

$$
E_{\mathrm{M} 3}=\frac{E_{\mathrm{M} 2}}{1+E_{\mathrm{M} 2} / 2 E_{\mathrm{A}}}
$$




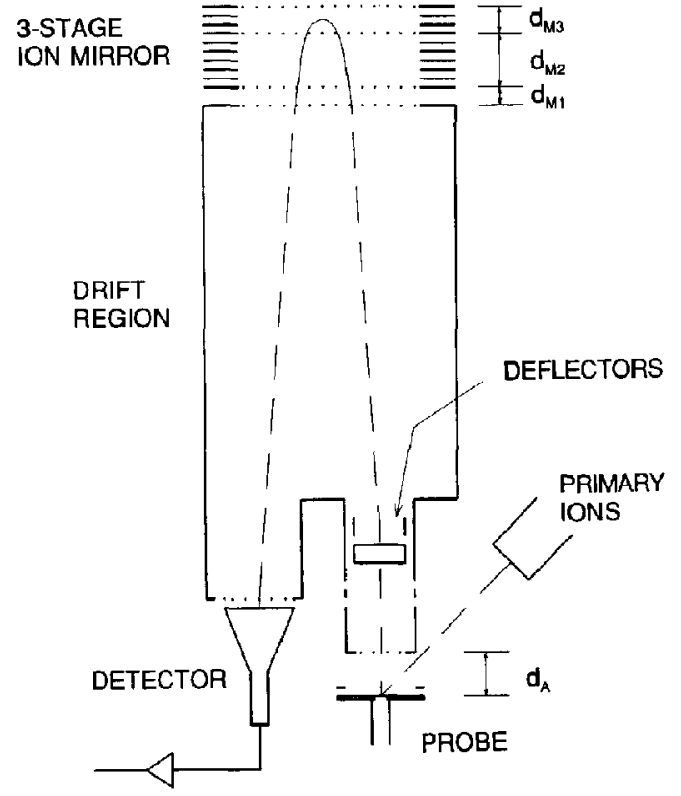

Figure 5. Schematic of experimental apparatus for TOF measurements. Pertinent parameters are listed in Table 2.

is very similar to eq 15 for the new two-stage mirror. As with the new two-stage design, the magnitude of the last mirror electric field is determined by the magnitude of the conventional mirror reflecting field and the acceleration electric field. Similar plots as shown in Figures 2 and 3 can be derived for the conventional two-stage and new three-stage ion mirror. The results are negligibly different from those displayed in Figures 2 and 3 , hecause the second-order corrections provided by the three-stage mirror are not very large.

\section{Experimental}

A SIMS TOF mass spectrometer was constructed to test the theory for improved energy compensation discussed above. The mass spectrometer has a short drift path and a relatively large acceleration (or secondary ion extraction) distance to accentuate effects of the initial ion kinetic energy distribution in the acceleration region. The experimental apparatus is shown schematically in Figure 5. A commercially available two-stage ion mirror ( $R$. M. Jordan, Grass Valley, CA) was modified by shortening the overall length and adding an extra grid, which resulted in a three-stage mirror of the type shown in Figure $4 \mathrm{~b}$ and described above. Critical dimensions of the TOF apparatus are listed in Table 2 along with other pertinent experimental parameters.

The primary ion gun, obtained commercially (Phrasor Scientific, Duarte, CA), is a tetrode source that consists of a thermionic $\mathrm{Cs}^{+}$source, extraction, focus and ground electrode system. The $\mathrm{Cs}^{+}$ions are accelerated to $8 \mathrm{keV}$ and focused through a 1-mm-di-
Table 2. Experimental TOF mass spectrometer parameters

\begin{tabular}{lll}
\hline Parameter & \multicolumn{1}{c}{ Description } & Value \\
\hline$d_{\mathrm{A}}$ & Acceleration length & $2.50 \mathrm{~cm}$ \\
$L$ & Drift length & $58.4 \mathrm{~cm}$ \\
$d_{M 1}$ & First mirror stage length & $1.17 \mathrm{~cm}$ \\
$d_{M 2}$ & Second mirror stage length & $3.05 \mathrm{~cm}$ \\
$d_{M 3}$ & Third mirror stage length & $1.53 \mathrm{~cm}$ \\
$V_{\mathrm{A}}$ & Probe voltage & $200 \mathrm{~V}$ \\
$V_{\mathrm{L}}$ & Drift region voltage & $0 \mathrm{~V}$ \\
$V_{\mathrm{M} 1}$ & Mirror retarding grid voltage & $130 \mathrm{~V}$ \\
$V_{\mathrm{M} 2}$ & First mirror reflecting grid voltage & $195 \mathrm{~V}$ \\
$V_{\mathrm{M} 3}$ & Second mirror reflecting grid valtage & $224 \mathrm{~V}$ \\
\hline
\end{tabular}

ameter aperture approximately $26 \mathrm{~cm}$ from the exit of the gun. A double deflector system near the exit of the primary ion gun allows alignment of the primary beam with the aperture as well as the ability to achieve a short pulse of primary ions on the target. The target probe is approximately $5 \mathrm{~cm}$ from the collimation aperture, and the primary ion beam strikes the target at a $45^{\circ}$ angle of incidence. A 10-ns [full width at half maximum (FWHM)] pulse of primary ions was produced by deflecting the ion beam away from the aperture and then sweeping the beam across the aperture using a fast (25-ns rise time) high voltage $(1.0-\mathrm{kV})$ pulse from a GRX-1.5K-E Pulser (Directed Energy, Inc, Fort Collins, CO). The primary ion beam pulse was characterized by placing a dual-microchannel-plate detector with an impedance-matched anode (model FTD2001, Galileo Electro-Optics Corp., Sturbridge, MA) in the location of the target probe and recording the amplified signal with a LeCroy (Chestnut Ridge, NY) 7200A digital oscilloscope.

Samples were placed on the end of a retractable and replaceable $1.3-\mathrm{cm}$-diameter probe that was inserted into fixed position relative to the primary ion beam and secondary ion extraction grid. To extract secondary ions from the sample and accelerate them to their nominal drift energy for TOF mass analysis, the probe was floated at $200 \mathrm{~V}$ and the extraction grid and field-free drift region were held at ground potential. After acceleration, ions passed through a short einzel lens and two separate sets of orthogonal deflection plates. The einzel lens was used to focus the secondary ion beam onto the detector, and one set of deflectors was used to deflect the secondary ion beam at the angle necessary to strike the detector after being reflected by the ion mirror. The other set of deflectors was used to align the beam with the detector in the orthogonal direction.

Ion mirror voltages for optimum temporal focusing are listed in Table 2 . Voltages to the fringe field plates in the ion mirror were supplied through the resistor chain provided with the mirror. By using eq 19 , the optimum mirror electric field ratio was calculated to be $E_{\mathrm{M} 3}=(0.89) E_{\mathrm{M} 2}$. According to the theoretical development above, $V_{\mathrm{M} 2}$ should be chosen so that only 
ions with non-zero initial energy enter the third region of the mirror, that is, ions that have exactly $200-\mathrm{eV}$ drift energy turn around at the grid. The actual value used reflects the maximum angle (approximately $9^{\circ}$ ) introduced by the secondary ion deflection plates. Deflection reduces the $z$-component of velocity, and consequently the retarding and reflecting voltages must be reduced by $\cos ^{2}\left(9^{\circ}\right)$ factor. Reduction of mirror voltages by a $\cos ^{2} \theta$ also would be necessary if the entrance angle $\theta$ into the mirror were introduced by tilting the mirror axis with respect to the mean ion axis of the first drift region.

Secondary ions were finally accelerated to $-2.2 \mathrm{kV}$ across a 1-mm distance into the FTD-2001 detector (Galileo Electro-Optics Corp., Sturbridge, MA). The detector signal was amplified with an ORTEC (Oak Ridge, TN) 9305 preamplifier and recorded using a LeCroy $7200 \mathrm{~A}$ digital oscilloscope. The trigger used to pulse the primary ion beam was used as the start trigger for acquisition of each sweep of the digital oscilloscope. The trigger frequency was $500 \mathrm{~Hz}$ and each TOF spectrum acquired was comprised of 10,000 sweeps, averaged by the digital oscilloscope. Spectra were acquired for various $E_{\mathrm{M} 3} / E_{\mathrm{M} 2}$ ratios by changing the value of $V_{\mathrm{M} 3}$ for each spectrum.

Three types of samples were used to test the theory for a variety of mass-to-charge ratio ions. Approximately $2 \mu \mathrm{L}$ of saturated solutions of $\mathrm{Na} I$ and Cs I in distilled water were deposited onto probe tips and vacuum dried to provide sources of $\mathrm{Na}^{+}(m / z 23)$ and $\mathrm{Cs}^{+}(m / z$ 133) ions, respectively. Approximately $1 \mu \mathrm{L}$ of $\mathrm{LiCl}$ in $\mathrm{HCl}$ was deposited onto another probe tip and vacuum dried to provide a source of $\mathrm{Li}^{+}(m / z 7)$. Elemental species were chosen because they have large kinetic energy distributions from secondary ionization and they do not suffer from in-flight metastable decay.

\section{Results and Discussion}

Figure 6 displays the predicted difference in flight time for $\mathrm{Na}^{+}$ions $(m / z$ 23) in the TOF mass spectrometer as a function of initial energy. The case $E_{\mathrm{M} 3}=E_{\mathrm{M} 2}$ is identical to the conventional two-stage mirror; ions with higher $U_{\mathrm{i}}$ have shorter overall flight times for this configuration. As shown previously in Figure 2, this behavior is essentially entirely due to the flight time in the acceleration region. The middle curve demonstrates that at the predicted $E_{\mathrm{M} 3} / E_{\mathrm{M} 2}$ ratio of 0.89 for temporal focusing, the flight time is very nearly independent of iritial energy. If $E_{\mathrm{M} 3}$ is reduced further, ions with higher $U_{i}$ have longer predicted flight times - the mirror overcompensates for an initial kinetic energy distribution.

In Figure 7 are three experimental TOF spectra of $\mathrm{Na}^{+}$ions for the three cases used for the calculations shown in Figure 6. As predicted, when the conventional two-stage mirror configuration is used $\left(E_{\mathrm{M} 3}=\right.$ $E_{\mathrm{M} 2}$ ), the peak has a noticeable tail on the shorter flight time side; the effect is apparent in Figure 7a. When the

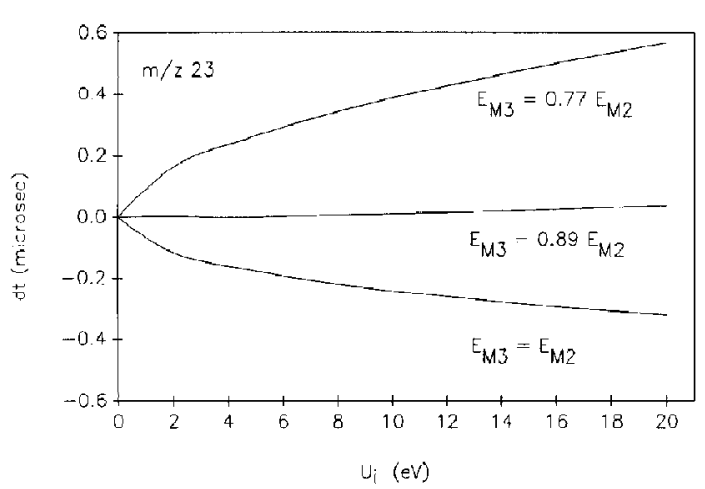

Figure 6. Predicted flight time differences for $\mathrm{Na}^{+}$ions as a function of initial energy. Calculations were based on actual dimensions of experimental apparatus for a nominal $200-\mathrm{eV}$ drift energy.

optimum field ratio (as calculated by using $\mathrm{Eq} 19$ ) is used, the TOF peak is very symmetric (Figure $7 \mathrm{~b}$ ). The peak also has a minimum FWHM and a maximum intensity for this configuration-the mass resolution is improved without sacrificing ion signal. The residual peak width is believed to arise primarily from fringe fields in the TOF mass spectrometer and the 10-ns duration of the primary ion beam pulse. Analysis of the secondary ion extraction, focusing, and deflection fields with SIMION [32] indicates that ionis away from the mean ion axis experience slightly different fields and therefore have slightly different flight times than ions traveling on axis. Figure $7 \mathfrak{c}$ demonstrates the effect of overcompensation predicted in Figure 6 for $E_{\mathrm{M} 3}$ values lower than optimum. The tail on the longer flight time side of the peak extends for nearly $0.5 \mu \mathrm{s}$.

Similar spectra were obtained for $\mathrm{Cs}^{+}$and $\mathrm{Li}^{+}$ions with very similar results. Minimum peak widths were found at very close to the optimum field ratio of 0.89 . Spectra also were obtained for $500-\mathrm{eV}$ nominal drift energy. Experimental parameters used for these measurements are found by multiplying all voltages in Table 2 (except the detector voltage) by a factor of 2.5 . Results from all of these spectra are displayed in Figure 8. Regardless of the mass-to-charge ratio or acceleration voltage, minimum peak widths were found at very close to the predicted field ratio. The effect of $E_{\mathrm{M} 3} / E_{\mathrm{MZ}}$ ratio on peak width appears to be stronger as the mass-to-charge ratio increases and acceleration voltage decreases. The weakest dependence was found for $m / z 23$ at $500 \mathrm{eV}$ (no data were acquired for $m / z 7$ at $500 \mathrm{eV}$ ), and the strongest dependence was found for $m / z 133$ at $200 \mathrm{eV}$.

For TOF mass spectrometers with small extraction distances and large acceleration voltages, effects of the acceleration region on mass resolution are small. It is not clear whether modification of the ion mirror as described above would result in an actual improvement in mass resolution in these situations. If a grid is used to define the terminal electric field, as suggested 

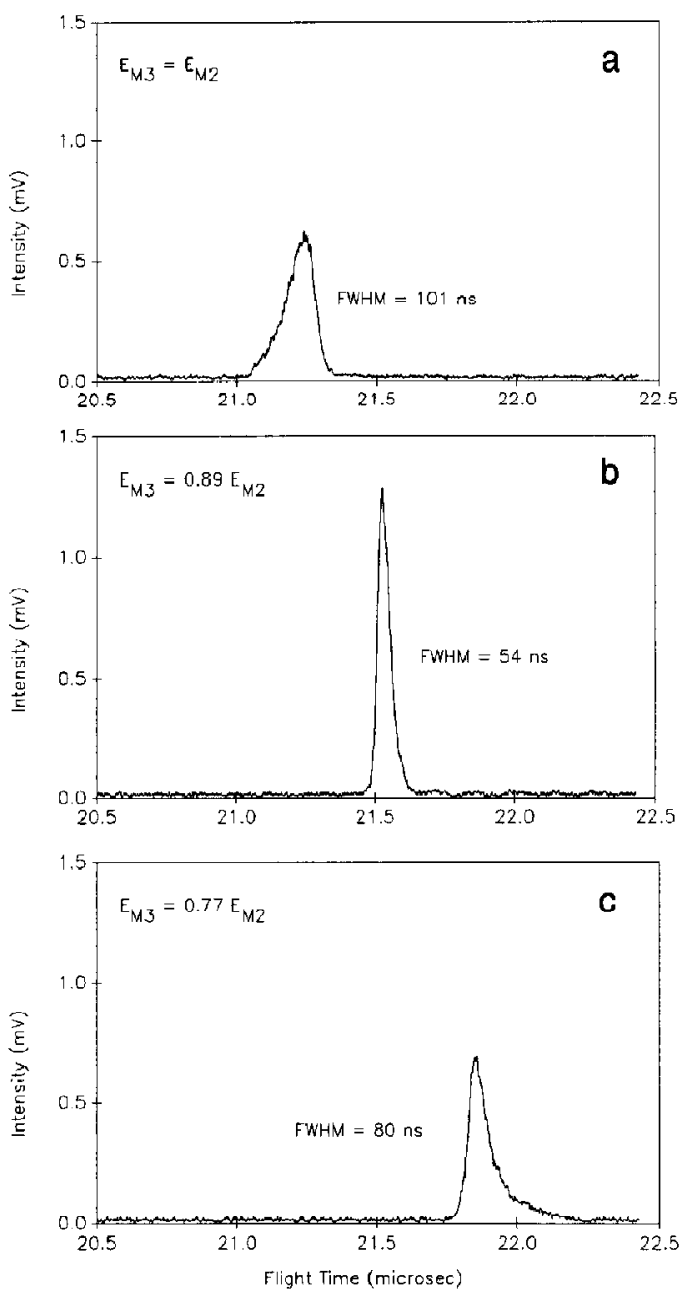

Figure 7. Experimental TOF spectra for $\mathrm{Na}^{+}$ions as a function of $E_{\mathrm{M} 3} / E_{\mathrm{M} 2}$ ratio for (a) conventional two-stage configuration, (b) optimum temporal focusing, and (c) overcompensation. All spectra were acquired for nominal 200-eV drift energy.

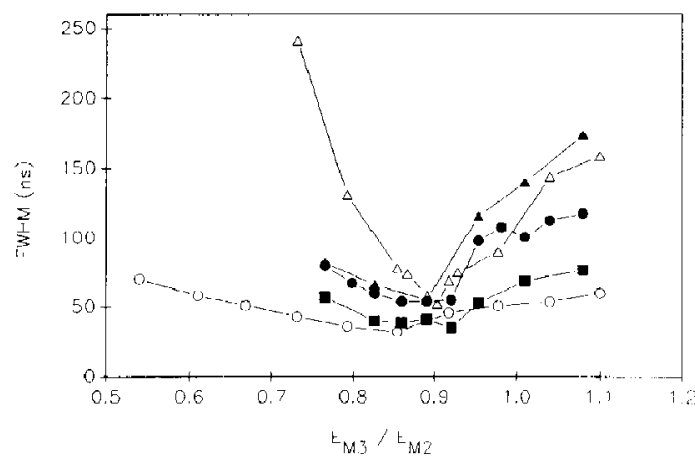

Figure 8. Plot of peak width as a function of mirror electric field ratio for (U) $\mathrm{Li}^{+}$at $200 \mathrm{eV}$, (৫) $\mathrm{Na}^{+}$at $200 \mathrm{eV},(0) \mathrm{Na}^{+}$at 500 $\mathrm{eV},(\triangle) \mathrm{Cs}^{+}$at $200 \mathrm{eV}$, and $(\Delta) \mathrm{Cs}^{+}$at $500 \mathrm{eV}$. herein, field distortions from the grid may cause a loss in mass resolution that is greater than the gain afforded by the new mirror configuration. Although the electric fields are not drastically different on either side of the grid, the ions have very low kinetic energy in the vicinity of the grid. For cases where the influence of the acceleration region is very small, it might be possible to omit the grid, but improve mass resolution by reducing the field at the end of the mirror in the same manner as one would if a grid had been installed. If the ions with nonzero initial kinetic energy experience a slightly reduced field at the end of the mirror, mass resolution may be increased with this technique, even though the field is not uniform.

Finally, the development in this paper does not deal with the problem of in-flight decay of molecular ions (either metastable or from collisions with grids and background gas) or kinetic energy defects. Mass changing processes can have a much more serious effect than kinetic energy distribution on mass resolution for reflectron TOF mass spectrometers, especially for analysis of very large biomolecules. In these cases, a modified ion mirror would serve little or no purpose. The technique described in this article compensates for a positive kinetic energy distribution, that is, ions are assumed to be formed at the surface of the sample probe. Recent measurements of kinetic energy defects of MALDI produced ions imply that these ions may be formed predominantly $10-100 \mu \mathrm{m}$ above the sample surface and/or may lose kinetic energy in the acceleration region from collisions in the dense plume created by the laser [35]. Although this energy compensation technique is not directly applicable to a kinetic energy defect, the magnitude of the energy defect could be significantly reduced by using a weak extraction field. The resulting increased effect of the positive kinetic energy distribution in the acceleration region could then be compensated for by use of a modified ion mirror.

\section{Conclusions}

The close correspondence of experimental performance of the three-stage ion mirror with its predicted performance gives us confidence that the mirror compensates for flight time spreads in the acceleration region of the TOF mass spectrometer. The ability to directly compensate for the acceleration and drift regions of the mass spectrometer provides much more flexibility for TOF surface desorption ion source design. The extraction grid can be placed much farther from the sample, which reduces memory effects from inadvertent sample sputtering and facilitates introduction of ionizing laser and ion beams. The new two- and three-stage mirrors also make high performance compact TOF mass spectrometers more feasible because the acceleration region no longer needs to be very small relative to the drift region, and acceleration voltages need not be high to minimize flight times in the acceleration region. 


\section{Acknowledgments}

This work was sponsored by the U.S. Department of Energy, Office of Research and Development (Experimental) and Office of Basic Energy Sciences (Theoretical), under Contract DE-AC05840R21400 with Martin Marietta Energy Systems, Inc.

\section{References}

1. Benninghoven, A.; Sichtermann, W. K. Aral. Chem. 1978, 50, 1180.

2. Steffens, P.; Neihuis, E.; Friese, T.; Greifendorf, D.; Benninghoven, A. I. Vac. Sci. Techrol. A 1985, 3, 1322.

3. Chait, B. T.; Standing, K. G. Int. I. Mass Spectrom. Ion Phys. 1981, 40, 185

4. Cotter, R. J. Anal. Chem. Acta 1987, 195, 45.

5. Karas, M.; Backmann, D.; Bahr, U.; Hillenkamp, F. Int. J. Mass Spectrom. Ion Process. 1987, 78, 53.

6. Hillenkamp, F.; Karas, M.; Beavis, R. C.; Chait, B. T. Anal. Chem. 1991, 63, 1193.

7. Macfarlane, R. D.; Torgerson, D. F. Science 1976, 191, 920; Int. I. Mass Spectrom. Ion Phys. 1976, 21, 81.

8. Della Negra, S.; Le Beyec, Y. Int. I. Mass Spectrom. Ion Process. 1984, 61, 21.

9. Brinkmalm, G.; Håkansson, P.; Kjelberg, J.; Demirev, P.; Sundqvist, B. U. R.; Ens, W. Int. J. Mass Spectrom. Ion Process. 1992, 114, 183.

10. Wiley, W. C.; McLaren, I. H. Rev. Sci. Instrum. 1955, $26,1150$.

11. Short, R. T.; O, C.-S.; Levin, J. C.; Sellin, I. A.; Liljeby, L.; Huldt, S.; Johansson, S.-E.; Nilsson, E.; Church, D. A. Phys. Rev. Lett. 1986, 56, 2614.

12. Levin, J. C.; Shart, R. T.; O, C..S.; Cederquist, H.; Elston, S. B.; Gibbons, J. P.; Sellin, I. A.; Schmidt-Böcking, H. Phys. Rev. A 1987, 36, 1649.

13. Karataev, V. I.; Mamyrin, B. A.; Shmikk, D. V. Zh. Tekh. Fiz. 1971, 41, 1498; Sov. Phys.-Tech. Phys. 1972, 16, 1177.

14. Mamyrin, B. A.; Karataev, D. V.; Schmikk, D. V. Sov. Phys. JETP 1973, 37, 45.

15. Mamyrin, B. A.; Shmikk, D. V. Sov. Phys. JETP 1979, 49(5), 762.

16. Tang, X.; Beavis, R.; Ens, W.; Lafortune, F.; Schueler, B.; Standing, K, G. Int. I. Mass Spectrom. Ion Process. 1988, 85, 43.
17. Niehuis, E.; Heller, T.; Feld, H.; Benninghoven, A. J. Vac. Sci. Technol. A 1987, 5, 1243.

18. Walter, K.; Boesl, U.; Schlag, E. W. Int. I. Mass Spectrom Ion Process. 1986, 71(3), 309.

19. Yang, M.; Reilly, J. P. Int. T. Mass Spectrom. Ion Process. 1987, 75, 209.

20. Frey, R.; Weiss, G.; Kaminski, H.; Schlag, E. W. Z. Naturforsch. A 1985, 40, 1349.

21. Grix, R.; Kutscher, R.; Li, G.; Grunner, U.; Wollnik, H. Rapid Commun. Mass Spectrom. 1988, 2, 83.

22. Wollnik, H.; Przewloka, M. Int. J. Mass Spectrom. Ion Process. 1990, 96, 267.

23. Bergmann, T.; Martin, T. P.; Schaber, H. Rev. Sci. Instrum. 1990, 67(10), 2592.

24. Kinsel, G. R.; Johnston, M. V. Int. I. Mass Spectrom. Ion Process. 1989, 9i, 157.

25. Yefchak, G. E.; Enke, C. G.; Holland, J. H. Int. I. Mass Spectrom. Ion Process. 1989, 87, 313.

26. Muga, M. L. Anal. Instrum. 1987, 16, 31.

27. Kinsel, G. R.; Mowry, C. D.; McKeown, P. J.; Johnston, M. V. Int. J. Mass Spectrom. Ion Process. 1991, 104, 35.

28. Standing, K. G.; Ens, W.; Tang, X. Proceedings of the International Conference on Instrumentation for Time-of-Flight Mass Spectrometry; Chestnut Ridge, NY, November 1992; p 9.

29. Todd, P. J.; Short, R. T.; Grimm, C. C.; Hotland, W. M.; Markey, S. P. Anal. Chem. 1992, 64, 1871.

30. Vestal, M. L. U. S. Patent Number 5,160,840, Time-of-Flight Analyzer and Method, Nov. 3, 1992.

31. Vestal, M. L.; Nelson, R. W. Proceedings of the 40th ASMS Conference on Mass Spectrometry and Allied Topics; Washington, DC, May 192; p 350.

32. Dahl, D. A.; Delmore, J. E. The SIMION PC/PS2 User's Manual, Version 4, Informal Report EGG-CS-7233, Rev. 2, Idaho Falls National Engineering Laboratory, 1988.

33. Tang, X. Ph.D. Dissertation, University of Manitoba, 1991.

34. Boesl, U.; Weindauf, R.; Schlag, E. W. Int. J. Mass Spectrom. Ion Process. 1992, 112, 121.

35. Zhou, J.; Erıs, W.; Standiry, K. G.; Verentchikuv, A. Rapid Commun. Mass Spectrom. 1992, 6, 671. 